Notfall Rettungsmed 2012 · 15:475-476

DOI 10.1007/s10049-011-1564-3

Online publiziert: 26. September 2012

(c) Springer-Verlag 2012

\author{
H.-R. Arntz ${ }^{1}$ B. Dirks ${ }^{2}$ \\ ${ }^{1}$ Medizinische Klinik II, Kardiologie und Pulmonologie, \\ Charite - Universitätsmedizin Berlin, Campus Benjamin Franklin, Berlin \\ ${ }^{2}$ Klinik für Anästhesiologie, Universitätsklinikum Ulm
}

\title{
Kontroversen in der Reanimatologie
}

Den Anlass des Wiener ERC-KongresKontroversen sind eine wesentliche Grundlage und Motivation wissenschaftlicher Arbeit. Sie fordern geradezu dazu auf, sie auf Basis eindeutiger Erkenntnisse aufzulösen. Die Reanimatologie mit ihrem schwierigen Umfeld, den zusammnehängenden ethischen Fragen und den extrem unterschiedlichen logistischen und sonstigen Randbedingungen ist erst in den letzten 10-15 Jahren ein Feld für Kontroversen geworden - und das mit steigender Tendenz. Nach der (Neu)-Beschreibung der Herzdruckmassage und Atemspende durch Kouwenhoven, Jude und Knickerbocker im Jahre 1960 mit Ergänzungen durch Safar und der Einführung der Defibrillation schienen die Probleme zunächst gelöst und die Welt war in Ordnung. Wissenschaftliche Studien, sowohl in der Klinik als auch prähospital, v. a. aber Tierexperimente ließen manche der scheinbar unverrückbar erscheinenden Grundlagen ins Wanken geraten und sind Hintergrund der zumeist bis heute ungelösten offenen Fragen und kontroverser Vorstellungen zum richtigen Vorgehen bei der kardiopulmonalen Reanimation. Nur wenige Fragen scheinen zumindest bisher relativ endgültig beantwortet. So ist inzwischen die herausragende Bedeutung der Herzdruckmassage bei der Reanimation zumal des Erwachsenen mit kardial bedingtem Keislaufstillstand akzeptiert. Andere Probleme wie z. B. das der grundsätzlichen Indikation bzw. Kontraindikation des Einsatzes von Vasopressoren, die Frage des am besten geeigneten Vasopressors sowie - wenn überhaupt - der optimale Einsatzzeitpunkt und die geeignete Dosis sind aktuell weiter denn je von einer Lösung entfernt. ses 2012 haben wir als geeignet betrachtet, einige der aktuell bestehenden und intensiv diskutierten Kontroversen in der Reanimatologie aufzugreifen und in einer Art Pro-Kontra-Darstellung im Leitthemenblock dieser Ausgabe von Nofall + Rettungsmedizin darzustellen. Wir sind hocherfreut, dass es uns gelungen ist, international anerkannte ausgesprochene Protagonisten als Autoren für die entsprechenden Themen zu gewinnen.

Ewy, Tucson, Arizona, USA und Koster aus Amsterdam, Niederlande diskutieren die heiß debattierte und sicher nicht endgültig beantwortete Frage nach der „Compression-only-“ gegenüber der „Compression-plus-Ventilation“-Strategie als grundlegendes Vorgehen bei der Reanimation. Wik, Oslo, Norwegen und Morrison, Toronto, Ontario, Canada hinterfragen die Effektivität einer Phase vorausgehender Thoraxkompressionen vor erster Rhythmusanalyse und Defibrillationsversuch bei Patienten mit einer „down time“ von einigen Minuten. Kern, Tucson, Arizona, USA gibt sich als starker Befürworter der routinemäßigen Koronarographie und evtl. PCI nach erfolgreicher Reanimation aus vermuteter kardialer Ursache zu erkennen, während Arntz aus Berlin an diesem grundsätzlichen Vorgehen erhebliche Zweifel anmeldet.

Wir sind uns sicher, mit diesen Themen und deren Darstellung sowohl das Interesse der Leserschaft getroffen zu haben als auch eine vertiefte Diskussion der offenen Fragen auslösen zu können und evtl. sogar die Anregung zu Untersuchungen zu geben, die zu einer Lösung der bestehenden Kontroversen beitragen kön- nen. Wir hoffen darüber hinaus, dass unsere Leserschaft auch in Anbetracht der Internationalität des anstehenden Kongresses und der Autoren akzeptiert, dass die Artikel überwiegend in englischer Sprache abgefasst sind und wünschen Ihnen intensive Anregung bei der Lektüre.

Ihre

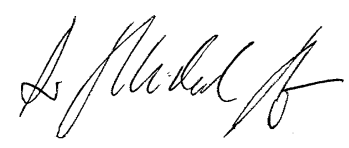

H.-R. Arntz

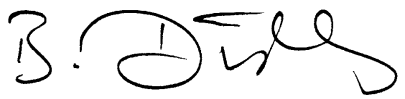

D. Dirks

\section{Korrespondenzadresse}

Prof. Dr. H.-R. Arntz

Medizinische Klinik II, Kardiologie und

Pulmonologie, Charite - Universitätsmedizin

Berlin, Campus Benjamin Franklin Hindenburgdamm 30, 12200 Berlin hans-richard.arntz@charite.de 


\section{Hier steht eine Anzeige.}

黛 Springer 